\title{
The humoral immune response to influenza vaccination in lung transplant patients
}

\author{
P.J. Mazzone*, S.B. Mossad", S.D. Mawhorter", A.C. Mehta*, R.J. Schilz*, J.R. Maurer*
}

The humoral immune response to influenza vaccination in lung transplant patients. P.J. Mazzone, S.B. Mossad, S.D. Mawhorter, A.C. Mehta, R.J. Schilz, J.R. Maurer. (C) ERS Journals Ltd 2001.

ABSTRACT: The purpose of this study was to evaluate the humoral immune response to influenza vaccination in lung transplant recipients.

Antibody levels to the three viral antigens included in the 1999-2000 trivalent influenza vaccine (A/Sydney/5/97-like (H3N2), A/Beijing262/95-like (H1N1), and B/Yamanashi/16/ 98) were measured before and 4 weeks postvaccination in 43 lung transplant recipients and 21 healthy adult controls. The ability to develop protective antibody levels, a serological response, and the magnitude of change in levels were assessed.

The humoral immune response to influenza vaccination was significantly lower in the transplant group for all three viral antigens. To A/Sydney, $95 \%$ of the control group and $40 \%$ of the transplant group developed protective levels $(\mathrm{p}=0.0009)$; to $\mathrm{A} / \mathrm{Beijing}, 71 \%$ of the control group and $30 \%$ of the transplant group developed protective levels $(\mathrm{p}=\mathbf{0 . 0 0 4 )}$; and to $\mathrm{B} /$ Yamanashi, $48 \%$ of the control group and $19 \%$ of the transplant group developed protective levels $(\mathrm{p}=\mathbf{0 . 0 2})$. Those receiving cyclosporine had lower antibody responses when compared to those receiving tacrolimus $(r=-0.3056, p=0.0463)$.

The humoral immune response to influenza vaccination in lung transplant recipients is poor. Lung transplant recipients receiving cyclosporine may have a lower antibody response than those receiving tacrolimus. Alternative prevention strategies may be needed. Eur Respir J 2001; 18: 971-976.
*Dept of Pulmonary and Critical Care Medicine, The Cleveland Clinic Foundation, and ${ }^{\#}$ Dept of Infectious Diseases, The Cleveland Clinic Foundation, Cleveland, OH, USA.

Correspondence: P.J. Mazone, Dept of Pulmonary and Critical Care Medicine, 9500 Euclid Ave A90, Cleveland, OH 44195 , USA.

Fax: 12164458160

Keywords: Antibodies

cyclosporine

influenza

lung transplantation

tacrolimus

vaccination

Received: February 282001

Accepted after revision July 102001
Infection with influenza viruses cause substantial morbidity and mortality each year. This is particularly true in older patients, those with chronic cardiovascular and pulmonary disease, and immunocompromised persons $[1,2]$. Patients receiving immunosuppressive drug therapy, such as solid organ transplant recipients, appear to be more susceptible to influenza infection, as well as more likely to experience related complications [3-7]. Several factors may predispose lung-transplant recipients to respiratory infections: 1) denervation of the transplanted lung(s) leading to a suppressed cough; 2) disruption of lymphatic drainage; 3) impaired mucociliary clearance in the transplanted lung; 4) presence of airway injury during periods of rejection; 5) coexisting pathology in the native lung of a single lung-transplant recipient; and 6) high levels of immunosuppression [8]. Due to the increased susceptibility and risk, prevention through annual influenza vaccination is recommended. However, since transplant patients are heavily immunosuppressed, the effectiveness of vaccination has been questioned.

Traditionally, studies of the immunogenicity of influenza vaccination have focused on the humoral response. The humoral response to vaccination has been assessed by determining the percentage of recipients who develop protective antibody titres (i.e. $\geqslant 1: 40$ ) or who develop a serological response (i.e. $\geqslant$ four-fold increase in titre) [9-12]. Nonmedication related factors have been identified that may alter the humoral response to the vaccine in a normal host. In particular, it tends to be more immunogenic in individuals who have had prior antigenic experience (i.e. infection, prior immunization) [10-13] and less immunogenic in the elderly [14], those with endstage renal disease, and those with advanced human immunodeficiency virus (HIV) infection $[9,10,13,15$, 16]. In studies of the humoral response in transplant populations, the use of mycophenolate mofetil [17] or cyclosporine $[18,19]$ and decreased allograft function $[11,19,20]$ were all predictors of decreased antibody response in some, but not other studies. Prior immunization and younger age were predictors of increased antibody response [10, 21]. In studies that used booster doses, these were not found to increase antibody production $[3,10,13,19,21]$.

The goal of this study was to assess the humoral immune response to influenza vaccination in lungtransplant recipients. To the best of the authors' knowledge, there are no previous studies focusing on vaccine immunogenicity in lung-transplant recipients.

\section{Patients and methods}

\section{Study subjects}

Lung-transplant recipients, who were at least 3 months post-transplantation, were recruited from 
the Cleveland Clinic Foundation (CCF) lung transplant programme. Exclusion criteria included: egg allergy, acute febrile illness, or active treatment for an acute rejection episode. Healthy adult controls (aged $\geqslant 18$ yrs) were recruited from the employees of the CCF. Exclusion criteria for controls included: egg allergy, a known immune function impairment, a known major organ disease, or use of immunosuppressive medication. The study was approved by the Institutional Review Board of the CCF and all study participants gave informed consent.

\section{Study design}

This study was a prospective observational cohort study designed to determine the humoral immune response to influenza vaccination in lung-transplant patients. A group of healthy adults served as the control population. Venous blood samples were collected prevaccination and 4 weeks postvaccination from both groups. All study participants received the 1999-2000 trivalent influenza vaccine containing A/Sydney/5/97-like (H3N2), A/Beijing/262/95-like (H1N1), and B/Yamanashi/16/98 antigens (WyethLederle, Marietta, PA, USA - Lot\# 4008203). All blood samples were tested for antibody titres to these three antigens.

\section{Methods}

Blood samples for haemagglutinin inhibition assays had their serum separated and stored frozen $\left(-20^{\circ} \mathrm{C}\right)$ immediately after collection. They were sent frozen as a batch to the Glennan Centre laboratory at Eastern Virginia Medical School (Norfolk, VA, USA) for assay at the conclusion of the study.

Haemagglutination assays were performed using haemagglutinin antigens representing the strains of virus contained in the vaccine. Haemagglutination inhibition was performed as previously described [22] using two-fold dilutions of serum from one in 10 to one in 1,024. Titres of less than one in 10 were calculated as one in five. Geometric mean titres were calculated.

\section{Analysis}

Outcome measures included: 1) the absolute antibody titres pre- and postvaccination; 2) the absolute change in titre from pre- to postvaccination; 3) the percentage of vaccine recipients who developed protective antibody levels (defined as titres $\geqslant 1: 40$ ); and 4) the percentage of vaccine recipients who were able to seroconvert (defined as a $\geqslant$ four-fold increase in titres). Independent variables collected and analysed included age, sex, time since transplantation, immunosuppressive drug use and levels, absolute lymphocyte count, prior vaccination status, serum creatinine, total imunoglobulin (Ig)-G levels, and acute-rejection episodes. Acute-rejection episodes required pathology graded at $\mathrm{A} 2 \mathrm{~B} 0$ or higher.
Differences in demographic variables between the control group and the transplant group were tested (Wilcoxon rank-sum test for continuous variables and Chi-squared for categorical variables). Differences in baseline variables were adjusted for in all subsequent tests.

Antibody titres were subjected to an analysis of variance (ANOVA for log transformed continuous variables and logistic regression for categorical variables) to see whether there were significant differences between the control group and the transplant group pre- and postvaccination. The Wilcoxon sign-rank test was used to test for within-group differences to determine whether or not the postvalue was significantly different from the prevalue.

The number of antigens to which seroconversion occurred (minimum zero, maximum three) and the number of antigens to which a protective antibody level developed (minimum zero, maximum three) were calculated separately. A Spearman correlation was performed on each of the independent variables listed earlier. These calculated values were used to assess for associations between the independent variables and the antibody responses within the transplant group.

The median, $25 \%$ quartile, and $75 \%$ quartile or percentages were computed as summary statistics, with the exception of antibody titres, which are presented as geometric means with $95 \%$ confidence intervals (CI). Changes in antibody titres are presented with medians and interquartile ranges (IQR) because of potentially "zero" changes. Significant differences were assessed with $\mathrm{p}<0.05$. Sample size was calculated with a power of $90 \%$ to detect a difference in antibody response assuming a control group response of $80 \%$ and a transplant group response of $40 \%$.

\section{Results}

Lung-transplant recipients $(n=43)$ and healthy adult controls $(n=21)$ enrolled and completed the study. The median age (yrs) of the groups differed, 51 (IQR 43-60 yrs) in the transplant group and 42 (IQR $41-44$ yrs $)$ in the control group $(p=0.008)$. The percentage of study participants who were male $(56 \%$ of the transplant group and $43 \%$ of the control, $\mathrm{p}=0.33)$, and the percentage receiving the previous years' vaccine $(100 \%$ of the transplant group and $86 \%$ of the control, $\mathrm{p}=0.52$ ) did not differ between the groups. The characteristics of the transplant group are shown in table 1 .

Antibody titres were available for all participants. The geometric mean titres were significantly lower in the transplant group both pre- and postvaccination (table 2). The ability to develop protective antibody levels was significantly less in the transplant group for all viral antigens (fig. 1). Ninety-five per cent of the control group and $40 \%$ of the transplant group developed protective levels to A/Sydney $(\mathrm{p}=0.0009)$; $71 \%$ of the control group and $30 \%$ of the transplant group developed protective levels to A/Beijing $(p=0.004)$; and $48 \%$ of the control group and $19 \%$ of the transplant group developed protective levels to 
Table 1. - Characteristics of transplant recipients studied

\begin{tabular}{lc}
\hline Male & $24(56)$ \\
Age yrs & $51(43-60)$ \\
Disease & $15(35)$ \\
COPD & $11(26)$ \\
A1AT & $6(14)$ \\
IPF & $4(9)$ \\
PPH & $7(15)$ \\
Other & $17(8-61)$ \\
Time since transplantation months & $26(60)$ \\
Single lung transplant & $23(53)$ \\
Cyclosporine use & $386(299-496)$ \\
Cyclosporine level $\mathrm{ng} \cdot \mathrm{mL}^{-1}$ & $20(47)$ \\
Tacrolimus use & $10.8(8.7-15.35)$ \\
Tacrolimus level ng $\cdot \mathrm{mL}^{-1}$ & $29(67)$ \\
Azathioprine use & $7(16)$ \\
Mycophenelate use & $10(7.5-15)$ \\
Prednisone daily dose $\mathrm{mg}_{\text {Absolute lymphocyte } \mathrm{count} \mathrm{K} \cdot \mathrm{uL}}$ & $1.24(0.94-1.65)$ \\
IgG level mg $\cdot \mathrm{dL}^{-1}$ & $577(445-753)$ \\
Creatinine $\mu \mathrm{mol} \cdot \mathrm{L}^{-1}$ & $150(114-220)$ \\
\hline
\end{tabular}

Data are presented as $\mathrm{n}(\%)$ or mean (interquartile range). COPD: chronic obstructive pulmonary disease; A1AT: alpha1-antitrypsin; IPF: idiopathic pulmonary fibrosis; PPH: primary pulmonary hypertension; IgG: immunoglobulin-G.

$\mathrm{B} /$ Yamanashi $(\mathrm{p}=0.02)$. The seroconversion rate was significantly lower for both influenza A antigens (fig. 2). To A/Sydney, 52\% of the control group and $26 \%$ of the transplant group seroconverted $(\mathrm{p}=0.009)$; to A/Beijing, $57 \%$ of the control group and 19\% of the transplant group seroconverted $(\mathrm{p}=0.004)$; and to $\mathrm{B} /$ Yamanashi, $24 \%$ of the control group and $12 \%$ of the transplant group seroconverted $(\mathrm{p}=0.16)$. The absolute increase in titre was lower in the transplant group for all three viral antigens (fig. 3). The median (IQR) change for the control and transplant groups respectively were: A/Sydney: 73 (26-240) versus 10 (0-33), $\mathrm{p}=0.0004 ; \mathrm{A} /$ Beijing: $30(12-120)$ versus 4 $(0-15), \quad \mathrm{p}=0.0002$; and to $\mathrm{B} /$ Yamanashi $8 \quad(0-40)$ versus $0(0-10), \mathrm{p}=0.01$.

Independent variables were analysed for correlations with the number of antigens to which an individual showed seroconversion, as well as the number of antigens to which there was a protective postvaccination level. There was a statistically significant inverse correlation between the use of cyclosporine (versus tacrolimus) and the antibody response $(r=-0.3115, p=0.0420$ for seroconversion, and $r=-0.3056, p=0.0463$ for the development of protective levels). There was a trend towards a decreased antibody response with higher cyclosporine levels within the group that was receiving cyclosporine

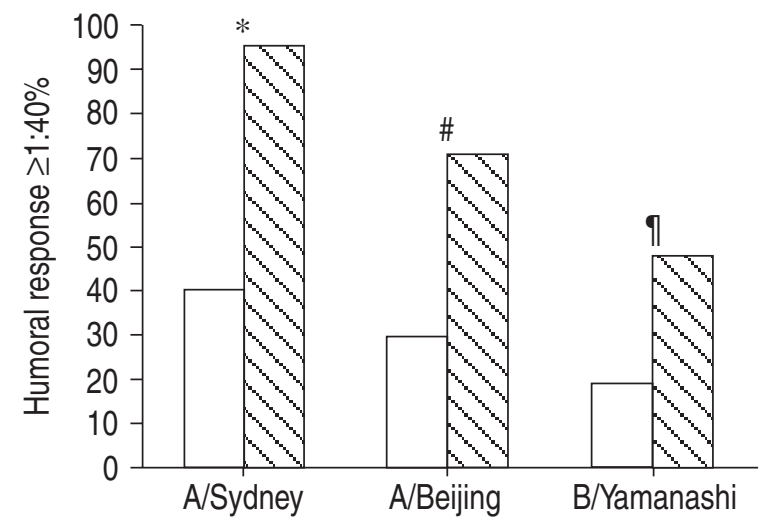

Fig. 1. - Development of protective antibody levels. $\square$ : transplant; $\mathbb{\mathbb { V }}$ : control. *: $\mathrm{p}=0.0009 ; \#$ : $\mathrm{p}=0.004 ; \boldsymbol{\uparrow}: \mathrm{p}=0.0164$.

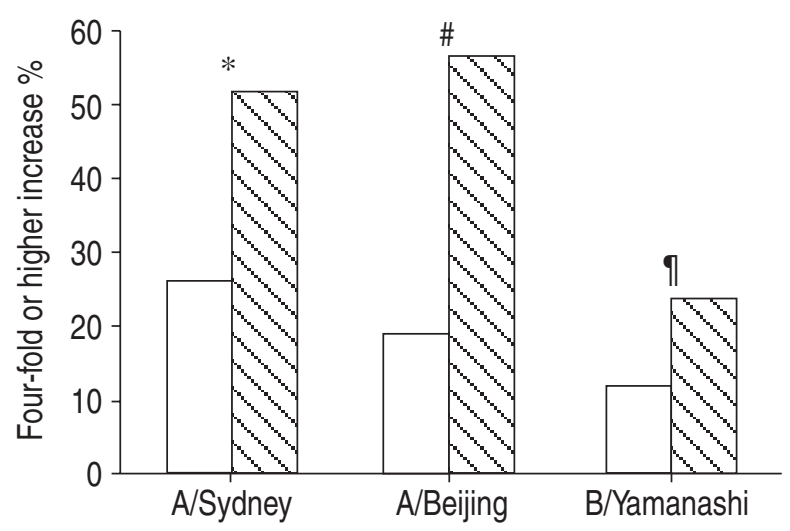

Fig. 2. - Development of serological responses. $\square$ : transplant; $\mathbb{\mathbb { }}$ : control. *: $\mathrm{p}=0.0085$; \#: $\mathrm{p}=0.0022 ; \boldsymbol{\uparrow}: \mathrm{p}=0.1582$.

( $r=-0.3834, p=0.0709$ for both antibody response measures). Five of the 43 transplant recipients developed a serological response to all three viral antigens. Each of these five were receiving tacrolimus (i.e. five out of 20 in the tacrolimus group, zero out of 23 in the cyclosporine group) (fig. 4). There were no significant differences between the group that received cyclosporine and the group that received tacrolimus amongst the other independent variables.

Correlations showing trends that did not reach statistical significance included: higher total IgG levels with better antibody responses $(r=0.278, p=0.07$ for seroconversion, and $\mathrm{r}=0.262, \mathrm{p}=0.089$ for the development of protective levels); the use of mycophenolate with decreased antibody responses $(r=-0.271, p=0.078$

Table 2.-Geometric mean (95\% confidence interval) antibody titres

\begin{tabular}{|c|c|c|c|c|c|c|}
\hline & \multicolumn{2}{|c|}{ A/Sydney } & \multicolumn{2}{|c|}{ A/Beijing } & \multicolumn{2}{|c|}{ B/Yamanashi } \\
\hline & Pre & Post & Pre & Post & Pre & Post \\
\hline Control & $39(24-62)$ & $157(91-272)$ & $15(9-25)$ & $81(43-153)$ & $17(12-25)$ & $34(22-52)$ \\
\hline Transplant & $16(13-19)$ & $33(24-46)$ & $9(7-11)$ & $16(12-23)$ & $11(9-13)$ & $16(12-20)$ \\
\hline $\mathrm{p}$-value & 0.0002 & $>0.0001$ & 0.04 & $<0.0001$ & 0.03 & 0.001 \\
\hline
\end{tabular}

$\mathrm{p}$-value analysis adjusted for age. 


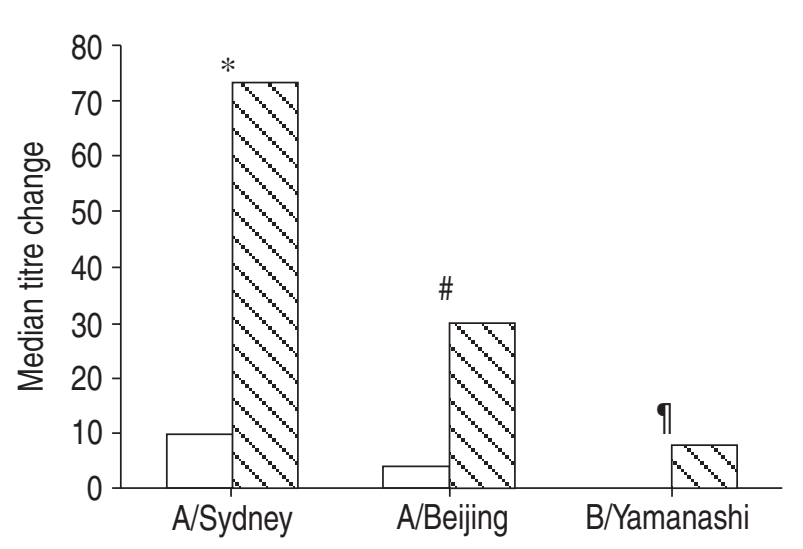

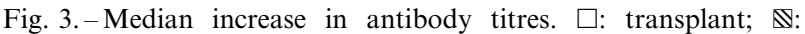
control. *: $\mathrm{p}=0.0004 ; \#: \mathrm{p}=0.0002 ; \uparrow: \mathrm{p}=0.01$.

for seroconversion, and $\mathrm{r}=-0.269, \mathrm{p}=0.081$ for the development of protective levels); and higher absolute lymphocyte counts with better antibody responses ( $\mathrm{r}=$ $0.242, p=0.118$ for seroconversion, and $r=0.23, p=0.13$ for the development of protective levels). There was no correlation between the antibody response to vaccination and the development of episodes of acute allograft rejection.

\section{Discussion}

It has been shown that there is a poor humoral immune response to influenza vaccination in lungtransplant recipients. Protective antibody titres developed in $19-40 \%$ of transplant-study participants to the antigens included in the vaccine. Only five of the $43(8.6 \%)$ developed protective levels to all three antigens. The pre- as well as the postvaccination antibody levels were lower in the transplant group, highlighting the immune-suppressed nature of this population.

The only statistically significant correlation among the independent variables was that cyclosporine use was associated with a smaller antibody response than tacrolimus use. Supporting this further was the trend

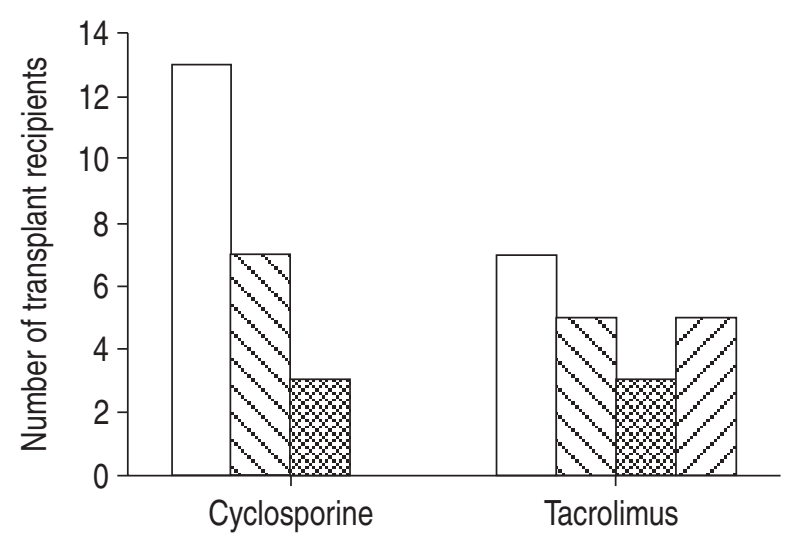

Fig. 4. - Number of antigens to which a serological response occurred. Cyclosporine patients, $\mathrm{n}=23$; Tacrolimus patients, $\mathrm{n}=20$. $\square$ : 0 antigens; $\mathbb{\nabla}: 1$ antigen; 2 antigens; $\mathbb{Z}: 3$ antigens. towards a decreased antibody response as the cyclosporine level increased. Other trends noted included lower antibody levels with the use of mycophenolate and greater antibody production with higher total IgG levels and higher absolute lymphocyte counts.

Several studies on the humoral immune response to influenza vaccination have been performed in other solid organ transplant populations.

In the renal transplant literature, studies on the immunogenicity of influenza vaccination have had different conclusions. Three studies have shown normal antibody responses when compared to controls $[10,23,24]$ while several others have described impaired immunogenicity [3, 17-20, 25]. In two of the studies with normal antibody production, prednisone and azathioprine were the only immunosuppressants used. There have been at least three studies that included a significant number of hearttransplant recipients $[3,13,26]$. All showed smaller humoral responses than healthy controls. Two studies in the paediatric liver transplant population showed relatively normal antibody production to vaccination $[10,21]$. One study that included adult liver transplant patients showed a decreased humoral response [3], while another showed antibody production equivalent to that of a control group [27]. Compared with the other solid-organ transplants, lung-transplant recipients tend to receive higher doses of immunosuppressive drugs and thus may be less likely to develop appropriate responses to vaccination.

It is unclear why the use of cyclosporine would lead to smaller humoral responses than tacrolimus as their mechanism of action is the same. Lungtransplant recipients may use one or the other drug depending upon their clinical course (e.g. episodes of acute rejection, neurotoxicity, nephrotoxicity, intolerance) or on institutional preferences. Two studies in the renal-transplant literature suggested that cyclosporine leads to decreased antibody production [18, 19]. In these studies, the transplant recipients not receiving cyclosporine were not receiving any calcineurin inhibitor. To the best of the authors' knowledge there are no previous studies comparing immunogenicity in patients receiving cyclosporine to those receiving tacrolimus. It is uncertain whether the findings in the present study represent a true difference in the effects of these drugs or a difference in the nature of the patients who end up receiving one or the other.

The major limitation to this study is the use of a surrogate marker of vaccine efficacy. The relatively small number of lung-transplant recipients available to study makes it difficult to have a large enough sample size to detect statistically and clinically significant differences in the development of influenzarelated illness. Although the humoral immune response to vaccination is a traditional marker of efficacy, it is known that some populations with decreased antibody production still receive clinically significant protection. For example, despite the decreased humoral response in the elderly, vaccination has been shown to prevent hospital admission and death by 72 and $87 \%$, respectively $[16,28]$. As the humoral response to vaccination does not always 
predict clinical protection against serious illness, recent studies in nontransplant populations have investigated the cellular response to influenza vaccination. Both T-helper and cytotoxic T-lymphocyte responses to influenza vaccination have been studied [29].

The results reported here should be generalizable to the population of lung-transplant recipients. It is more difficult to predict their relevance to other immunosuppressed populations. Other solid-organ transplants may have different levels of vaccine efficacy, as lungtransplant recipients tend to receive the highest levels of medical immunosuppression.

Future studies in this area will be important. Studies of the cellular immune response to vaccination will help elucidate the level and mechanism of protection. The development of more immunogenic vaccines, methods of delivery, or the use of chemoprevention are all areas of potential advances.

To conclude, the humoral immune response to influenza vaccination in lung-transplant patients is poor. Lung-transplant recipients receiving cyclosporine may have a decreased antibody response to influenza vaccination compared to those receiving tacrolimus. Further studies and alternative prevention strategies are needed.

\footnotetext{
Acknowledgements. The authors would like to thank the statistical contributions of $\mathrm{D}$. Nelson and his team. They are also indebted to the laboratory support provided by J.E. McElhaney and the laboratory at The Glennan Centre at Eastern Virginia Medical School, and B. Yen-Lieberman and the laboratory at The Cleveland Clinic Foundation.
}

\section{References}

1. Barker WH, Mullooly JP. Pneumonia and influenza deaths during epidemics: implications for prevention. Arch Intern Med 1982; 142: 85-89.

2. Mossad SB. Underused options for preventing and treating influenza. Clev Clin J Med 1999; 66: 19-23.

3. Blumberg EA, Albano C, Pruett $\mathrm{T}$, et al. The humunogenicity of influenza virus vaccine in solid organ transplant recipients. Clin Infect Dis 1996; 22: 295-302.

4. Ljungman $\mathrm{P}$, Andersson J, Aschan J, et al. Influenza A in immunocompromised patients. Clin Infect Dis 1993; 17: 244-247.

5. Beyer WE, Diepersloot RJ, Masurel N, Simoons ML, Weimar W. Double failure of influenza vaccination in a heart transplant patient. Transplantation 1987; 43: 319.

6. Mauch TJ, Bratton S, Myers T, Krane E, Gentry SR, Kashtan CE. Influenza B virus infection in pediatric solid organ transplant recipients. Pediatrics 1994; 94: 225-229.

7. Aschan J, Ringden O, Ljungman $\mathrm{P}$, Andersson $\mathrm{J}$, Lewensohn-Fuchs I, Forsgren M. Influenza B in transplant patients. Scand J Infect Dis 1989; 21: 349350 .

8. Wendt $\mathrm{CH}$. Community respiratory viruses: organ transplant recipients. Am J Med 1997; 102: 31-36.
9. Palache AM. Influenza vaccines - a reappraisal of their use. Drugs 1997; 54: 841-856.

10. Mauch TJ, Crouch NA, Freese DK, Braunlin EA, Dunn DL, Kashtan CE. Antibody response of pediatric solid organ transplant recipients to immunization against influenza virus. J Pediatr 1995; 127 : 957-960.

11. Pabico RC, Douglas G, Betts RF, McKenna BA, Freeman RB. Antibody response to influenza vaccination in renal transplant patients. Ann Intern Med 1976; 85: 431-436.

12. Potter CW. Determinants of immunity to influenza infection in man. Br Med Bull 1979; 35: 69-75.

13. Dengler TJ, Strnad N, Buhring I, et al. Differential immune response to influenza and pneumococcal vaccination in immunosuppressed patients after heart transplantation. Transplantation 1998; 664: 13401347.

14. Nichol KL, Margolis KL, Wuorenma J, von Sternberg $T$. The efficacy and cost effectiveness of vaccination against influenza among elderly persons living in the community. $N$ Engl J Med 1994; 331: 778784 .

15. Fiebach N, Beckett W. Prevention of respiratory infections in adults. Arch Intern Med 1994; 154: 25452557.

16. Gardner P, Schaffner W. Immunization of adults. $N$ Engl J Med 1993; 32: 1252-1258.

17. Smith KGC, Isbel NM, Catton MG, Leydon JA, Becker GJ, Walker RG. Suppression of the humoral immune response by mycophenolate mofetil. Nephrol Dial Transplant 1998; 13: 160-164.

18. Huang KL, Armstrong JA, Ho M. Antibody response after influenza immunization in renal transplant patients receiving cyclosporin $\mathrm{A}$ or azathioprine. Infect Immun 1983; 40: 421-424.

19. Versluis DJ, Beyer WE, Masurel N, Wenting GJ, Weimar W. Impairment of the immune response to influenza vaccination in renal transplant recipients by cyclosporine but not azathioprine. Transplantation 1981; 42: 376-379.

20. Kumar SS, Ventura AK, VanderWerf B. Influenza vaccination in renal transplant recipients. JAMA 1978; 239: 840-842.

21. Mack DR, Chartrand SA, Ruby EI, Antonson DL, Shaw BW, Heffron TG. Influenza vaccination following liver transplantation in children. Liver Transpl Surg 1996; 2: 431-437.

22. WHO Collaborating Center for Influenza, Biological Products Division. The Hemagglutination Inhibition Test for Influenza Viruses. Verson 31 revised, DHEW, PHS, CDC. Atlanta, GA, USA, Center for Infectious Disease, 1981; pp. 1-21.

23. Briggs WA, Rozek RJ, Migdal SD, et al. Influenza vaccination in kidney transplant recipients: cellular and humoral immune responses. Ann Intern Med 1980; 92: 471-477.

24. Carroll RNP, Marsh SD, O'Donoghue EPN, Breeze DC, Shackman R. Response to influenza vaccine by renal transplant patients. BMJ 1974; 2: 701-703.

25. Stiver HG, Graves P, Meiklejohn G, Schroter G, Eickhoff TC. Impaired serum antibody response to inactivated influenza $\mathrm{A}$ and $\mathrm{B}$ vaccine in renal transplant recipients. Infect Immun 1977; 16: 738-741.

26. Admon D, Engelhard D, Strauss N, Goldman N, Zakay-Rones Z. Antibody response to influenza 
immunization in patients after heart transplantation.

Vaccine 1997; 15: 1518-1522.

27. Burbach G, Bienzle U, Stark K, Rayes N, Neuhaus R, Serke S. Influenza vaccination in liver transplant recipients. Transplantation 1999; 67: 753-735.

28. Barker WH, Mullooly JP. Influenza vaccination of elderly persons: reduction in pneumonia and influenza hospitalizations and deaths. JAMA 1980; 244: 25472549.

29. McElhaney JE, Pinkoski MJ, Au D, Lechelt KE, Bleackley RC, Meneilly GS. Helper and cytotoxic $\mathrm{T}$ lymphocyte responses to influenza vaccination in healthy compared to diabetic elderly. Vaccine 1996; 14: $539-544$. 